Stefan Kubica, Hagen Ringshausen, Jörg Reiff-Stephan, Marius Schlingelhof (Hrsg.) 2. Automobil Symposium Wildau:

Tagungsband Technische Hochschule Wildau 2017

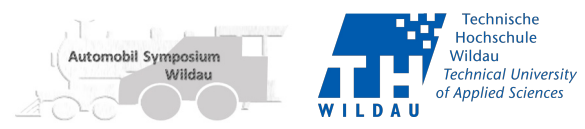

\title{
Digitales Entwickeln am Beispiel der Engineering-Cloud
}

\section{Zusammenfassung}

Die Entwicklungsabteilungen der Automobilbranche müssen sich heutzutage großen Herausforderungen stellen. In Zeiten von enormen Kosten- und Zeitdruck gilt es Gesamtsysteme, Baugruppen oder Einzelkomponenten wie z. B. Airbags, Rückfahrkameras, Türschlossoder Sitzsysteme möglichst ressourcenschonend und effizient zu entwickeln bzw. zu optimieren. Hinzu kommen Faktoren wie Leistungsfähigkeit, Energieeffizienz, Qualität, Zuverlässigkeit und positive Umwelteigenschaften, die über den Erfolg eines Produkts entscheiden. Damit Ingenieure diesen Anforderungen gerecht werden können, müssen leistungsstarke Ressourcen und Infrastruktur bereitstehen, die auf einer agilen IT-Struktur basieren.

\section{Produktentwicklungs- prozess}

Für die effiziente Produktentwicklung sind neueste Methoden erforderlich. In der Konstruktion hat sich insbesondere die numerische Simulation als Standardwerkzeug etabliert. Computer-AidedEngineering (CAE) prüft konstruktive Ideen bereits in der Planungsphase. Während der Designer zu Beginn mit dem Bleistift die Form des Produkts vor- gibt, erzeugt der Konstrukteur im Anschluss das 3D-Modell am Rechner, genannt Computer-Aided-Design (CAD). Schließlich lässt der Ingenieur die Physik auf das 3D-Modell einwirken. Dafür werden dem Fahrzeug zunächst sämtliche Materialien inklusive deren physikalische Materialeigenschaften zugewiesen. Beim anschließenden Meshing wird eine gegebene Oberfläche oder ein gegebenes Raumvolumen durch eine Menge kleinerer, meist sehr einfacher Elemente angenähert (approximiert). Das so entstehende Gitter ist eine vereinfachte Beschreibung der Fläche oder des Körpers, welches dann beispielsweise für weitergehende Berechnungen genutzt werden kann, etwa mittels der FiniteElemente-Methode (FEM). In der Entwicklung und Optimierung von automobilen Produkten werden vor allem CSM (Computational Structural Mechanics)- und CFD (Computational Fluid Dynamics)-Berechnungen durch-geführt, um Problemstellungen aus Struktur und Strömung sowie deren Wechselwirkung zu untersuchen. Der Bereich Noise, Vibration, Harshness (NVH) zur Berechnung von Ge-räuschen, Vibrationen und Rauheit ist erst in jüngster Zeit neu in die Prozesskette eingegliedert worden. 


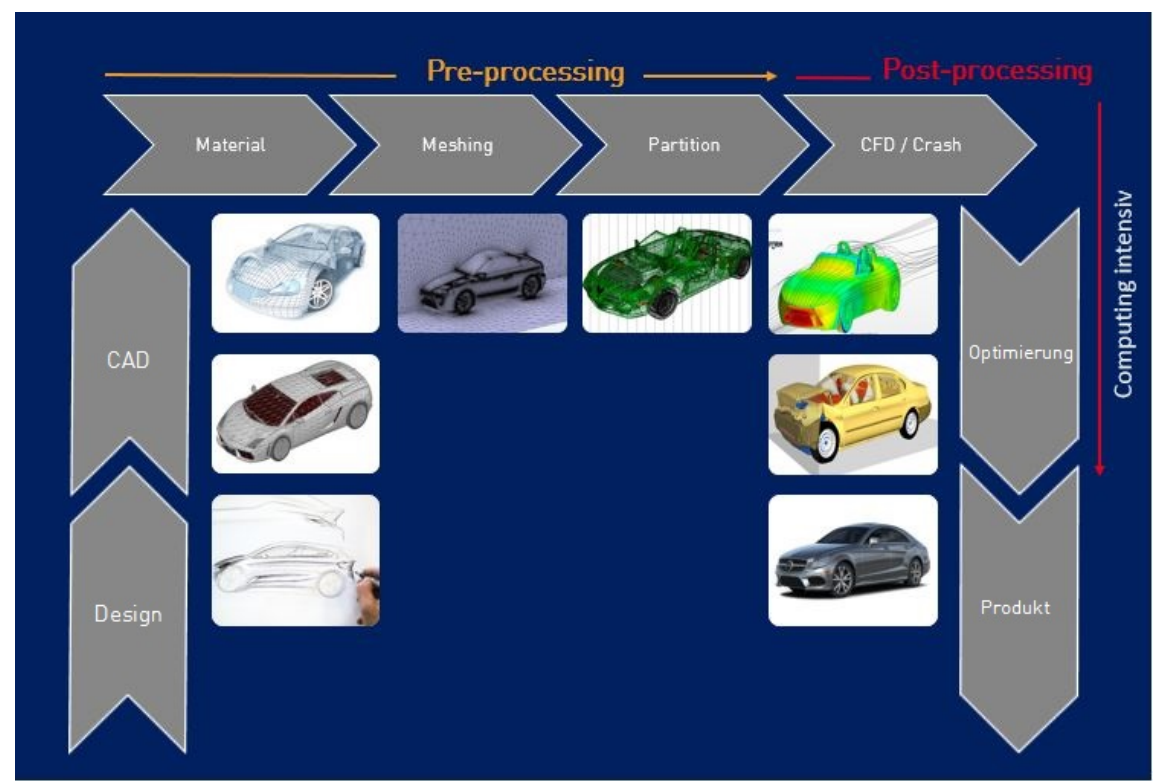

Abbildung 1: Produktentwicklungsprozess (Quelle: CPU 24/7 GmbH)

Der Rechenaufwand während der Entwicklung mittels Desktop-PC oder Produktentwicklungsphase ist immens. Leistungsstarke Rechenressourcen für den Erhalt von schnellen und genauen Simulationsergebnissen sind demzufolge unabdingbar. Parallel dazu wächst der Bedarf an Rechenleistung ungebremst, die Rechenressourcen werden immer leistungsstärker und universelle Simulationssoftware in Verbindung mit 3D-Visualisierungsgrogrammen verfügbarer. Laut einer aktuellen Marktstudie der Intersect360 Research verzeichnet der aggregierte High Performance Computing (HPC) Markt derzeit ein jährliches Wachstum von 5,2\% bis 2020 . Dafür sind mitunter verkürzte Produktentwicklungszeiten sowie die steigende Komplexität von neuen Produkten verantwortlich. Indes gewinnt die Auslagerung der Rechenkapazitäten oder Cloud-Nutzung in der simulationsgetriebenen Produktentwicklung und -optimierung - im Vergleich zur digitalen „on-premise" - immer mehr an Bedeutung. Denn nicht jedes Unternehmen ist in der Lage, solche Ressourcen angemessen zur Verfügung zu stellen oder diese - sofern überhaupt vorhanden kontinuierlich und effektiv auszunutzen. Neben Automotive sehen sich auch Branchen wie Marine \& Offshore, Transportation, Aerospace sowie Oil \& Gas mit limitierten Ressourcen und fehlender Agilität konfrontiert.

Sobald im Unternehmen die Entscheidung für eine Cloud-Lösung fällt, weil beispielsweise das interne Cluster für aufkommende Projekte nicht mehr ausreicht, muss zunächst eine geeignete Cloud-Lösung definiert werden. Diese muss auf die Bedarfe des Unternehmens sowie auf die Auslastung der bestehenden Ressourcen angepasst werden. Hier haben sich im Wesentlichen drei unterschiedliche Nutzungsmodelle herauskristallisiert. 


\section{Nutzungsmodelle der Engineering Cloud}

\subsection{Hybride Cloud-Lösung}

Das Feedback der Automotive-Ingenieure aus Gesprächen mit CPU 24/7 zeigt, dass sich durchschnittlich zwei bis dreimal pro Jahr sogenannte Spitzenlasten einstellen, bei denen unternehmensinterne Kapazitäten nicht mehr ausreichen, um richtungsweisende Projekte voranzutreiben oder fertigzustellen. Für die Abdeckung solcher unabsehbaren Projektspitzenlasten bedienen sich Automobilzulieferer im Rahmen des Outsourcings der Variante „CloudBursting“. Hierbei wird die Grundlast über die Inhouse-IT abgedeckt, Spitzenlasten werden vom Cloud-Dienstleister abgefangen. Mithilfe externer Kapazitäten werden fortan Queues re- duziert oder beseitigt, Ergebnisse schneller geliefert und Ingenieuren die Möglichkeit gegeben, mehr Simulationen simultan durchzuführen. So kann einer kostenintensiven Aufstockung der internen Rechenressourcen entgegengewirkt werden, während die volle Auslastung der bereits vorhandenen gesichert ist. Andersherum können bei einer schwachen Projektauslastung externe Rechenressourcen unkompliziert herunterskaliert oder auch abgeschaltet werden. Agilität und eine hohe Verfügbarkeit von Rechenkapazitäten des HPCCloud-Provider entscheiden über den Erfolg eines Outsourcings. Sofern dieser individuell auf die Bedarfe und Projekte des Kunden eingeht und die Infrastruktur entsprechend anpasst, ist der signifikante Unterschied in der Gewinnung von Simulationsergebnissen unwiderlegbar.

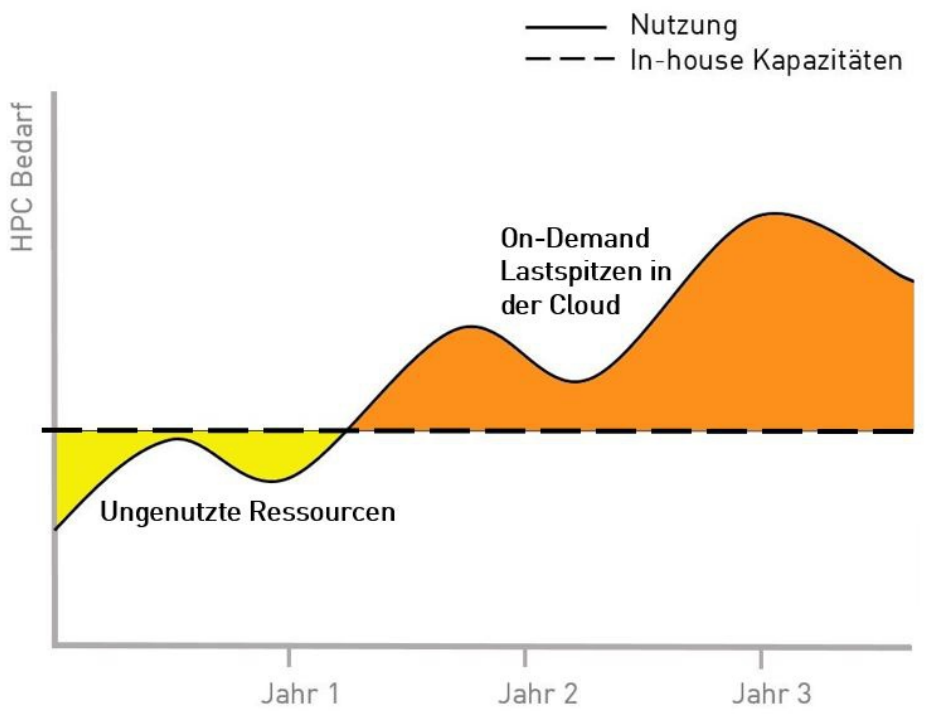

Abbildung 2: Cloud-Bursting (Quelle: CPU 24/7 GmbH) 


\subsection{Vollständiges Outsourcing}

Das Outsourcing der Gesamtstruktur auf stets aktuelle Architekturen wird im CAE-Bereich analog zu anderen CloudServices immer mehr zur anerkannten Alternative zum Inhouse-Betrieb. Bereits heute rechnen vereinzelte Abteilungen vollständig in der Cloud. Die Übernahme der Gesamtstruktur bleibt demzufolge nur noch eine Frage der Zeit.

Immerhin verhilft der vollständige Übergang in die Cloud Unternehmen zu erheblichen Kosten- und Zeiteinsparungen. Anschaffungskosten sowie Folgekosten für Hardware, Implementierungszeiten, Betriebskosten und überlastete Rechner entfallen. Ergebnisse werden aufgrund leistungsstärkerer Rechenressourcen schneller erzielt und Lizenzen effizienter ausgenutzt.

Daneben ermöglicht die CAE Enterprise Cloud-Lösung eine zentrale, standortunabhängige Nutzung von Projektdaten, dessen Bedarf durch die wachsende kollaborative Produktentwicklung/-optimierung stetig ansteigt. Denn schon längst ist das Zugreifen auf eine homogene und performante HPC-Infrastruktur von unterschiedlichen Entwicklungsabteilungen in verschiedenen Ländern keine Zukunftstheorie mehr, sondern wird bereits von unzähligen Unternehmen gelebt. Bei der Wahl des richtigen HPC-Cloud-Providers ist jedoch Vorsicht geboten, hier empfiehlt sich vorab eine umfangreiche und intensive Recherche. Nur ein vertrauenswürdiger Anbieter kann den vollen Schutz der sensiblen Entwicklungsdaten und des damit verbundenen geistigen Eigentums garantieren. Unumgänglich in der industriellen Forschung \& Entwicklung sind hierbei unter anderem eine dedizierte und private Infrastruktur mit individuellen Sicherheitsvereinbarungen.

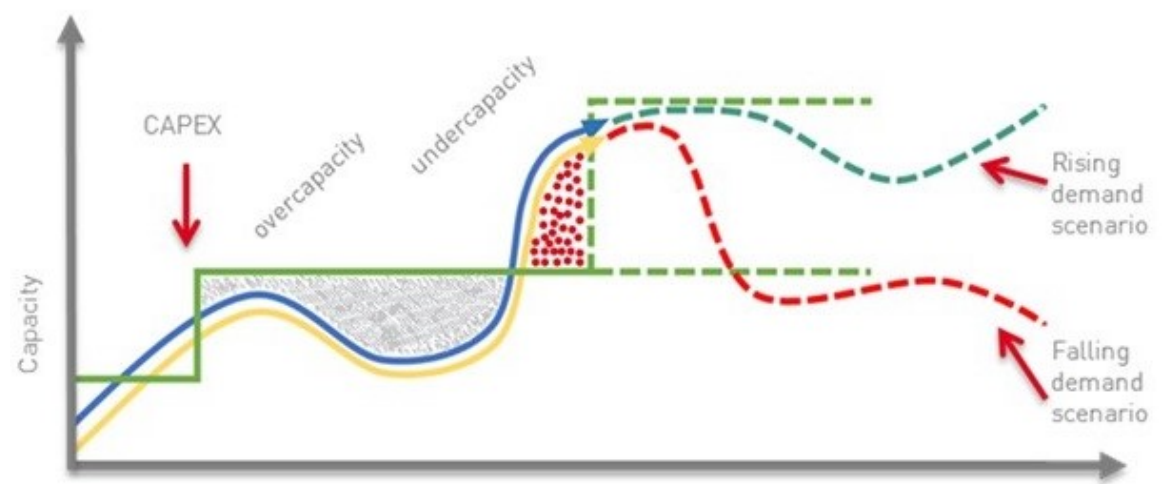

Time

Abbildung 3: Darstellung der verschiedenen Outsourcing-Modelle (Quelle: Wolfgang Gentzsch, UberCloud)24/7 GmbH) 


\subsection{Single Users/ Job Outsourcing}

Für kleine Ingenieursbüros oder kleine und mittelständige Unternehmen (KMU) ist die Nutzung von OutsourcingKonzepten ebenso rentabel. Dort wird standardmäßig an der Workstation gearbeitet und meist sind keinerlei oder nur eingeschränkte eigene Kapazitäten vorhanden. Ein Großteil der Ingenieure sieht sich aber mit vereinzelten komplexen Aufgabenstellungen konfrontiert, die mit gängigen Desktop-PCs innerhalb eines akzeptablen Zeitraums mit ausreichender Genauigkeit und Qualität nicht mehr lösbar sind. Ein Beispiel hierfür sind Berechnungen mit transienten Daten, bei denen Objekte untersucht werden, deren Zustände sich im Verlauf der Zeit verändern. Die zusätzlich differenzierte Betrachtung einer zeitlichen Komponente in einer instationären Berechnung erfordert eine höhere Rechenleistung, um letztendlich alle physikalischen Effekte auflösen zu können. Tests im Automobilbereich haben gezeigt, dass transiente Berechnungen auf HPC-Cloud-Ressourcen der aktuellen Generation um den Faktor fünf bis sechs schneller liefen im Vergleich zur Inhouse-Workstation.

\section{Aktuelle Herausforderungen}

Doch so fortschrittlich die Technologie sein mag, einige Herausforderungen in der Umsetzung gibt es dennoch. Die Ergebnisse der Umfrage „Top 5 Cloud Challenges of CPU 24/7" Customers der CPU 24/7 ergeben, dass die größten Herausforderungen aus Sicht der Ingenieure insbesondere in der Datensicherheit gesehen werden, dicht gefolgt von der Lizenzproblematik der Softwaregeber mit ihren z.T. noch sehr starren, inkompatiblen Lizenzmodellen und der fortschreitenden Globalisierung. In der Produktentwicklung sind Unternehmen von diversen Abstimmungen untereinander abhängig. Hierbei gilt es den Transfer großer Datenmengen zu ermöglichen und die damit verbundene Produktivität sicherzustellen. Weitere Herausforderungen gilt es in puncto Anpassbarkeit der internen Prozesse zu bewältigen, Service Level Agreements und Support sowie letztendlich nicht vorhersagbare Kosten, was z.T. an der intransparenten Preisgestaltung einiger HPC-Anbieter liegt.

Insbesondere Automobilzulieferer, die zumeist den Vorgaben der OEMs unterliegen, benötigen sehr umfassend durchdachte Cloud-Lösungen, um auch ihnen diese Art der Ressourcennutzung zu ermöglichen. Im Dialog zwischen Kunde und $\mathrm{CAE}$ as a Service-Provider gibt es bereits verschiedene „Best-Practice“-Ansätze, die sich jeweils in die drei Bereiche Zugang (Sicherheit, Performance), HPCTechnik (Remote Visualisierung, Interconnect, Storage, RZ-Standort) und operative Prozesse (Abrechnung, SLAs, Support, Consulting, Lizenzen) einordnen lassen und sich teilweise bedingen und aufeinander aufbauen.

\section{Marktbeispiel}

Im Falle eines global führenden deutschen Automobilzulieferers mit einer CAE-Abteilung von über 100 Berechnungsingenieuren hat sich CloudBursting als ein erfolgreich umgesetztes Outsourcing-Modell erwiesen.

Stark wachsende Geschäftsbereiche sowie die steigende Nachfrage der Automobilhersteller brachten eine massive Überlastung der hausinternen Rechenressourcen mit sich. Der Bedarf an computergestützten Produktsimulationen wuchs binnen drei Jahren um $30 \%$. Um 
auch weiterhin unter der Berücksichtigung von verkürzten Produktentwicklungszeiten kontinuierlich qualitative Produkte liefern zu können, bedurfte es einer Lösung für die schwer planbaren Lastspitzen. Anstatt erneut in Hardware, Software und Know-how zu investieren, entschied sich der Automobilzulieferer dazu, künftig aufkommende Projekte in der Engineering-Cloud von CPU 24/7 zu rechnen. Innerhalb kürzester Zeit wurde eine dedizierte und skalierbare Infrastruktur aufgebaut, die es mehreren Nutzern an verschiedenen Standorten ermöglicht, kollaborativ Produkte zu entwickeln. Die bereits vorhandenen Lizenzen des Zulieferers konnten dank der intensiven Kooperation zwischen dem HPC-Cloud-Provider CPU 24/7 und dem Softwarehersteller LS-DYNA geradewegs in den Prozess miteingebunden werden. Des Weiteren wurden individuelle Sicherheits- und ServiceLevel-Vereinbarungen getroffen, um die Geheimhaltung sowie Leistung von CPU 24/7 sicherzustellen.

Mithilfe externer Rechenkapazitäten hat sich der global agierende Automobilzulieferer die Möglichkeit geschaffen, am hart umkämpften Automobilmarkt weiterhin konkurrenzfähig zu bleiben. Die flexible und skalierbare Rechenleistung erlaubt dem Kunden, in Zukunft umgehend auf die veränderten Gegebenheiten des sich rasch entwickelnden Marktes der Automobilbranche, reagieren zu können.

\section{Fazit}

Der Großteil der Ingenieure, die mit Simulationssoftware arbeiten, führen Simulationen noch immer am DesktopPC durch. Dies wird aufgrund fortschreitender Technologie verbunden mit höherer Datenlast sowie der Internationalisierung von Projekten schon bald nicht mehr möglich sein. Folglich werden Ingenieure im Rahmen der digitalen Entwicklung nach und nach den Weg in die Cloud suchen. Attraktive Modelle, wie beispielsweise pay-per-use werden die Entwicklung des Marktes weiter begünstigen. Darüber hinaus sind gefestigte Partnerschaften zwischen HPCCloud-Anbietern mit Softwareherstellern maßgebend. Diese ermöglichen die Mitnahme von bestehenden Lizenzen in die Cloud sowie minuten- oder stundengenaue Abrechnungsmodelle, die exakt dem Bedarf und der Nutzung des Kunden entsprechen. Hauptargument für das Fortbestehen der Outsourcing-Modelle bleiben weiterhin die wirtschaftlichen und servicetechnischen Vorteile, wie z. B. Kosteneffizienz und die Möglichkeit bei Bedarf auf einen enormen HPCRessourcenpool zuzugreifen ohne Sicherheitseinbußen hinnehmen zu müssen. Aus diesem Grund wird sich Outsourcing früher oder später auch in hochspezialisierten Wirtschaftszweigen, wie z. B. der Forschung und Entwicklung durchsetzen.

\section{Literaturverzeichnis}

[SWS16]

Addison Snell, Christopher G. Willard, Laura Segervall: Worldwide High Performance Computing (HPC) 2016 Total Market Model and 2016-2020 Forecast. Intersect360 RESEARCH, 2016.

[HT16]

Alexander Heine, Jens Tamm: Top 5 Cloud Challenges of CPU 24/7 Customers. CPU 24/7 $\mathrm{GmbH}, 2016$.

[ID16]

IDC Research, Inc.: IDC HPC Pulse Survey 2016. www.idc.com. Abruf am 15.02.2017. 
[AN16]

ANSYS, Inc.: ANSYS DIMENSIONS Magazine, Summer 2016. http://resource.ansys.com/staticassets/ANSYS/staticassets/resourcelibrary/article/DIMENSIONS-AA-D-Summer-2016.pdf. Abruf am 15.02.2017.

(C) 10 BY NC ND $\begin{aligned} & \text { Dieser Beitrag ist unter der } \\ & \text { Creative-Commons-Lizenz }\end{aligned}$ 